\title{
High-Resolution and Analytical TEM Investigation of Space Radiation Processing Effects in Primitive Solar System Materials and Airless Planetary Surface Environments
}

\author{
R. Christoffersen ${ }^{1}$, L. P. Keller ${ }^{2}$, Z. Rahman ${ }^{1}$, C. Dukes ${ }^{3}$, R. Baragiola ${ }^{3}$ \\ ${ }^{1}$ ESCG/Jacobs Technology, Houston, TX 77258-8447 USA \\ ${ }^{2}$ Mail Code KR, ARES Directorate, NASA Johnson Space Center, Houston, TX 77058 USA \\ ${ }^{3}$ Laboratory for Atomic and Surface Physics, University of Virginia, Charlottesville, VA.
}

Energetic ions present in the diverse plasma conditions in space play a significant role in the formation and modification of solid phases found in environments ranging from the interstellar medium (ISM) to the surfaces of airless bodies such as asteroids and the Moon. These effects are often referred to as space radiation processing, a term that encompasses changes induced in natural space-exposed materials that may be only structural, such as in radiation-induced amorphization, or may involve ion-induced nanoscale to microscale chemical changes, as occurs in preferential sputtering and ion-beam mixing. Ion sputtering in general may also be responsible for partial or complete erosion of space exposed materials, in some instances possibly bringing about the complete destruction of free-floating solid grains in the ISM or in circumstellar nebular dust clouds.

We report here on two examples of the application of high-resolution and analytical transmission electron microscopy (TEM) to problems in space radiation processing. The first problem concerns the role of space radiation processing in controlling the overall fate of Fe sulfides as hosts for sulfur in the ISM [1]. The second problem concerns the known, but as yet poorly quantified, role of space radiation processing in lunar space weathering [2].

Whereas Fe sulfides are detected around evolved stars, and must be a grain component that is injected into the ISM along with abundant silicates, there is little astronomical evidence for solid sulfide grains in the ISM [2]. This suggests that unlike silicates, solid sulfides may be destroyed somewhere on their route to the ISM, possibly by some element of space radiation processing. As a first step in understanding the response of sulfides to space radiation processing relative to silicates, we used the IVEM-Tandem high-resolution TEM at Argonne National Laboratory [3] to perform insitu ion irradiation experiments on pyrrhotite $\left(\mathrm{Fe}_{1-\mathrm{x}} \mathrm{S}\right)$, troilite $(\mathrm{FeS})$, and for comparison forsterite ( $\left.\mathrm{FO}_{92}\right)$ and enstatite $\left(\mathrm{MgSiO}_{3}\right)$. Using $1.0 \mathrm{MeV} \mathrm{Kr}^{++}$ions with a high potential for structural damage by nuclear elastic collisions, we found the sulfides remained crystalline up to an experimentally practical maximum ion dose equivalent to 26 displacements-per-atom (DPA) (Fig. 1). This was two orders of magnitude higher than the DPA levels at which we found the silicates to become completely amorphous. While pyrrhotite retained its short-range crystalline substructure up to the 26 DPA level, its longer-range vacancy-ordered superstructure was lost at much lower DPA, equivalent to that at which olivine and enstatite become amorphous (Fig. 1). This suggests that space radiation conditions sufficient to amorphize olivine and enstatite in circumstellar and interstellar environments would convert co-existing pyrrhotite to its disordered structural form, thereby changing magnetic and possibly other properties that determine how pyrrhotite will behave in these environments.

Based on our sulfide amorphization findings, we extended our experiments to measure total ion sputtering rates of troilite (FeS) compared to silicates, work that focuses more directly on sulfide 
grain destruction in space radiation environments. We used an FEI Quanta 600 3D dual-beam focused ion beam (FIB) instrument to sputter sharp grain boundaries between troilite and enstatite or forsterite in sections of the Vigarano CV3 chondrite. After sputtering with $30 \mathrm{kV} \mathrm{Ga}+$ ions, we measured the relative depths of the sputtered pits produced in the adjacent phases by SEM and fieldemission STEM imaging. The FeS/enstatite pit depth ratios were measured at 4.2 and 3.9, and energy dispersive X-ray (EDX) spectrum imaging showed significant preferential sputtering removal of S from the troilite surface, forming a 5-9 nm thick surface layer of Fe metal. Our results indicate that FeS grains produced in evolved stars and injected into the ISM should be destroyed more rapidly than crystalline silicates. The relative of rate destruction suggested by our experiments is, however, not fast enough to remove all hope of finding interstellar sulfides in very primitive meteoritic materials, such as interplanetary dust particles.

Within the solar system itself, space radiation processing, mostly from solar wind ions, can have a major altering effect on the surface chemistry, structural state, and optical reflectance of regolith grains on the Moon and other airless bodies. We are currently investigating the detailed effects of ion irradiation on the surface properties of lunar grains through a combined X-ray photoelectron spectroscopy (XPS) and field-emission transmission electron (FE-STEM) study of experimentally ion irradiated lunar soil as well as key lunar soil minerals. On the monolayer depth scales probed by XPS, in-situ irradiation with both $4 \mathrm{keV} \mathrm{He}^{+}$ions progressively converts surface Fe on mature lunar soils from a +3 oxidation state caused by terrestrial exposure, to a metallic state ( $\mathrm{Fe}^{0}$ ) likely characteristic of lunar conditions. In-situ XPS ion irradiation finds this same radiation-induced surface reduction in pristine Fe-bearing lunar minerals such as ilmenite (Fig. 2). FIB cross-sections of these irradiated samples studied by field-emission STEM imaging and X-ray compositional mapping find that the surface reduction reaches well below the surface monolayer scale, extending over $100 \mathrm{~nm}$ into the sample (Fig. 2). Within this $100 \mathrm{~nm}$ region, the original ilmenite is significantly re-processed to contain a porous metallic Fe outer layer, with an implanted-He "bubble" layer below. The microstructure reflects complex changes in the Fe/Ti ratio as a function of depth relative to the original crystal. The results shed light on the ability of solar wind ions, acting without contribution from other processes, to drive a significant subset of the microstructural and chemical changes occurring in the lunar regolith in response to space weathering.

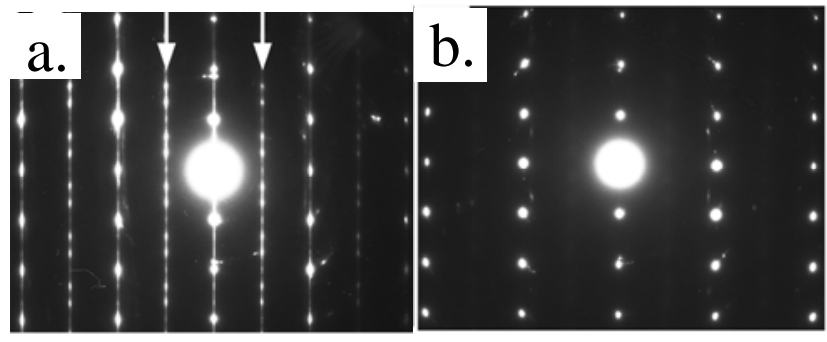

Fig. 1. Selected-area [010] electron diffraction patterns for 6C pyrrhotite polytype prior to (a) and after (b) $1 \mathrm{MeV} \mathrm{Kr}{ }^{+}$irradiation to $6 \times 10^{15}$ ions $/ \mathrm{cm}^{2}$

\section{References:}
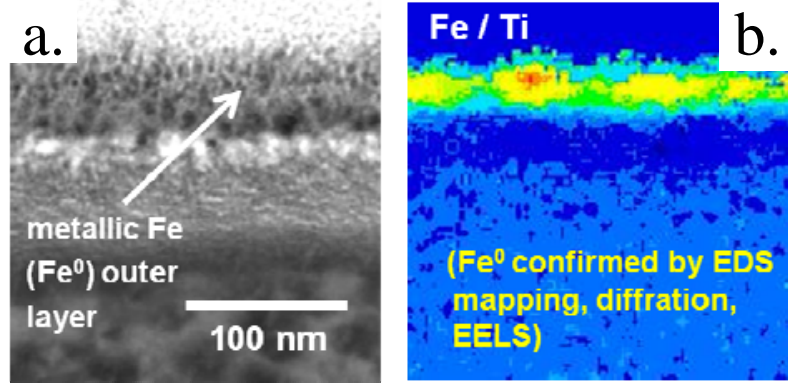

Fig.2. Bright-field STEM (a) and EDX compositional spectrum element ratio image (b) of outer 100 $\mathrm{nm}$ of ilmenite irradiated to $3 \times 10^{17} \mathrm{He}^{+}$ions $/ \mathrm{cm}^{2}$

[1] Keller, L. P., et al., Nature, v. 417, pp. 148-150

[2] Hapke, B., Journal of Geophysical Research, v. 106, pp. 10,039-10,073

[3] Kirk M. A., et al., Microscopy Research and Technique v. 72, pp. 182-186. 